\title{
ACUTE DEMYELINATING DISEASE COMPLICATING HERPES ZOSTER
}

\author{
BY \\ DOUGLAS McAlPINE,* Y. KUROIWA, Y. TOYOKURA, and S. ARAKI $\dagger$ \\ From the Third Department of Internal Medicine, the Medical School, University of Tokyo, and \\ the Department of Neuropsychiatry, Kumamoto University, Kyushu, Japan
}

Judging by the literature on the subject of the central nervous complications of herpes zoster, demyelination seems rare as a sequel. Up till 1945 Gordon and Tucker could find no recorded case in the few necropsy reports on encephalitis or myelitis complicating this infection. Nor have we been able to trace any proven case in the recent literature. This seems all the more remarkable since an early stage of a perivenous demyelinating encephalomyelitis is by no means a rare complication of varicella (Miller, Stanton, and Gibbons, 1956).

In the following two cases there were clinical grounds for believing that a demyelinating type of reaction was present.

\section{Case Reports}

Case 1.-T. Sano, a woman, aged 25, was admitted to the Third Department of Internal Medicine, Tokyo University, on March 16, 1956, under the care of Professor S. Okinaka. The following history was obtained. In the middle of February, 1956, a widespread herpetic eruption appeared, extending from the right costal margin upwards to the neck and involving the right arm. Two weeks later she noticed urgency and frequency of micturition. On March 12 numbness was felt around the lower ribs and quickly spread upwards to the chest and arms and downwards to the abdomen and lower limbs, which by February 14 had become weak. On the next day she developed urinary and rectal incontinence. When admitted on March 16, she was dyspnoeic. The neck was slightly stiff. The cranial nerves were normal. All four limbs were markedly weak and a sensory level at C.4 was obtained. The deep reflexes were depressed whilst both plantar responses were extensor. The cerebrospinal fluid contained 26 cells per c.mm. and $40 \mathrm{mg}$. per $100 \mathrm{ml}$. of protein.

A diagnosis of acute myelitis due to the virus of herpes zoster was made. She was treated with prednisolone, A.C.T.H., and acromycin. After 10 days, power had

* Present address: The Institute of Clinical Research, The Middlesex Hospital Medical School, London, W.1.

IIn receipt of grants from the National Institute of Neurological Diseases and Blindness, Bethesda 14, Maryland. returned to the limbs and at the end of a further five $\overparen{\overparen{D}}$ days sensation was normal, except in the left hand. Sphincter control was regained by the end of six weeks. क On May 17 she was discharged fully recovered.

First Relapse.-Early in December, 1956, she com- $\overrightarrow{\vec{H}}$ plained of giddiness, vomiting, and numbness of the $\omega^{\omega}$ left hand. The symptoms passed off after a few days but returned again on December 19 with hiccough. Prednisolone was given without effect. Three days before readmission on December 19 double incontinence $N$ recurred.

Examination showed hyperaemia of the optic disces, $\vec{N}$ well marked lateral nystagmus, hyperaesthesia, anf 0 weakness of the left upper limb, bilateral cerebellar $\frac{}{3}$ ataxia, and exaggeration of all deep reflexes, left mo⿷匚⿳亠口冋 than right.

The diagnosis now seemed to lie between disseminate्व: encephalomyelitis and multiple sclerosis. The respons $\vec{\varphi}$ to prednisolone, $10 \mathrm{mg}$. daily, was satisfactory. December 25 incontinence had cleared up and other symptoms had improved. By January 16, 1957, the only abnormal signs were nystagmus and sensory disturbance in the left hand. Prednisolone was discontinued.

Second Relapse.-Early in February, 1957, she again became ataxic and incontinent. Prednisolone was re-started and by the end of the month she had begun to improve. On April 14, when the drug was discontinued, all signs had cleared except nystagmus and sensory loss in the left hand.

Third Relapse.-On April 21, 1957, incontinence? returned with headache and generalized itching. On April 27 knee and ankle jerks were all diminished. Prednisolone, $20 \mathrm{mg}$., was started again and within five $\dot{0}$ days sphincter control had improved. The dose of 3 prednisolone was gradually reduced, except for a few days when incontinence returned. The drug was finally의 stopped at the beginning of October.

Fourth Relapse.-Ten days after the discontinuation of prednisolone, incontinence returned and was accom- $\rightarrow$ panied by mild fever, abdominal discomfort, and neuralgic pains which spread to the chest and upper extremities $N$ with some weakness of the hands. Prednisolone was at. once resumed and was continued in a dose of $7.5 \mathrm{mg} . N$ By the end of May, 1958, 5,457 mg. had been given.

Apart from a return of mild bladder symptoms for $a_{0}^{0}$ 
fortnight in June, 1958, she had no relapse till December of that year. Prednisolone was discontinued on November $30,1958$.

Case 2.-R. Nakano, a high school girl, aged 14, was apparently in good health until January 15, 1958, when she noticed a rash over the right upper chest. Two days later she felt tired but on the following day went to a cinema. During the performance she saw double and by the next morning her family noticed that she squinted. She consulted an ophthalmologist who found bilateral paresis of the internal rectus muscle. During the next few days vision deteriorated in the left eye. Both hands, the abdomen, and lower limbs felt numb, the right leg became weak, and she was unsteady when walking.

She was admitted to the Neuropsychiatric Department of Kumamoto University under the care of Professor K. Miyagawa on January 24. Temperature was normal. She was drowsy but normally orientated and cooperated well in the examination. Slight neck stiffness was present but she denied headache. A crusted herpetic eruption was seen in the distribution of the right third dorsal segment. The fundi were normal, but with the left eye she could only count fingers, and the left pupil responded sluggishly to light. There was paresis of conjugate movement to the left and of both internal recti with impairment of upward movement of both eyes. Nystagmus was present to the right and vertically. The corneal reflexes were brisk and sensation was normal on the face. A slight right facial weakness of peripheral type was present. The remaining cranial nerves were normal.

In the upper limbs both hand grips were weak, left more than right, fine movements were clumsy and a marked degree of cerebellar ataxia was present on finger-nose testing. Hypaesthesia was noted in the fingers of both hands with a mild degree of postural loss and faulty two-point discrimination. Hyperpathia was present over segments C. 2 and 3 and D. 2 and 3 with impairment of all forms of sensation below D.4 and gross postural loss in the toes of both feet. The abdominal reflexes were absent. There was slight loss of power in both lower limbs, particularly the right. Deep reflexes were depressed in the upper but exaggerated in the lower limbs, with the exception of the right knee jerk which was depressed. Both plantar responses were extensor.

Since the clinical picture suggested an acute demyelinating process, treatment with cortisone, $25 \mathrm{mg}$. four hourly, was begun.

On January 30 , the patient was more alert. Neck stiffness was less marked. Vision in the left eye showed perception of light only; right eye 0.4 (normal 1.2). There was complete paresis on conjugate movement to the left. Hand grips were both weaker, there was no voluntary movement of the right leg and early paresis in the left leg. Urine was being retained now. By January 31 , vision in the right eye and power in the left leg had further deteriorated; otherwise her condition was unchanged. Cortisone was increased to $200 \mathrm{mg}$. By February 3, both pupils were dilated, and she could only count fingers with the right eye; vision in the left eye was unchanged. Paresis of the upper limbs was more marked.
Postural loss in the joints of the right arm up to and including the elbow and in the left arm in the fingers and wrist joints was complete. Sensory level was now D.1 on the left side. Paraplegia was now complete except for feeble extension at the left knee. Postural loss was found in all joints in both lower limbs (hip joints not tested). Slight progression of symptoms during the previous three days was noted on February 5. The left upper limb reflexes were absent, with complete paraplegia and postural loss in the lower limbs. On February 8, prednisolone, $20 \mathrm{mg}$., was given in addition to cortisone, $150 \mathrm{mg}$.

On February 17, slight improvement was noted. Hand movements were appreciated with the left eye, and nystagmus was less marked. Both hand grips were stronger. The sensory level was now D.4. Power had slightly returned to the left knee but postural loss in both lower limbs remained complete. Cortisone was reduced to $100 \mathrm{mg}$. on February 14 .

Despite the development of a sacral decubitus the patient continued to improve. By the end of March she was able to read, ocular movements had returned to normal, and nystagmus was minimal. Apart from a mild degree of residual weakness in both hand grips and faulty two-point discrimination in the fingers, the upper limbs were normal. A sensory level was no longer found on the trunk. In the lower limbs a mild spastic paraparesis, superficial sensory loss, and postural loss at the ankles and toes remained. Bladder control returned. After débridement, the sacral sore began to heal. From February 14 the dose of cortisone was progressively decreased and finally stopped on March 2. Prednisolone, $10 \mathrm{mg}$., was continued for a further four days, the total amount given being $410 \mathrm{mg}$. and of cortisone $3,725 \mathrm{mg}$.

On May 21, 1958, examination by Dr. S. Seguchi showed the following:-Vision both eyes $1 \cdot 2$ (normal); no nystagmus; ocular movements were full. Power, reflexes, and sensation were normal in the upper limbs, without ataxia. Slight weakness of the right lower limb persisted; knee jerks were within normal limits but the right ankle jerk was exaggerated with unsustained clonus. Both plantar responses were early extensor. Bladder control was normal. The bedsore was nearly healed. The patient could walk several hundred metres without support.

\section{Discussion}

In the first of these two cases, two weeks after an attack of herpes zoster, frequency and urgency of micturition followed by an acute myelitis naturally suggested virus as a cause. Seven months later a severe relapse raised the question of a demyelinating disease, a diagnosis later confirmed by further relapses. In this patient any attempt to decide the issue as between acute disseminated encephalomyelitis and multiple sclerosis on purely clinical grounds must take into consideration a number of facts, some relating to the case itself and others to certain aspects of the natural history of these closely related conditions.

When relapses occur in the months or years after 
an attack of acute disseminated encephalomyelitis there can be three explanations (van Bogaert, 1950).

First, as a result of the original infection, the nervous system becomes sensitized and a subsequent exanthem or other type of infection may lead to a reactivation of the same lesion or may even cause a new and possibly fatal attack of encephalomyelitis. The second explanation assumes that the original illness represented the acute onset of multiple sclerosis. Lastly, in rare cases, it seems possible that multiple sclerosis may become grafted on to a previous attack of perivenous encephalomyelitis.

In our first case the latent interval of seven months before the first relapse, the clinical evidence of fresh lesions during this relapse in the form of giddiness, vomiting, and cerebellar ataxia, and lastly further relapses, seem to favour a diagnosis of multiple sclerosis, either grafted on to an attack of perivenous encephalomyelitis or arising $a b$ initio. However, it is also possible that the case falls into the first of van Bogaert's categories as an example of relapsing or chronic disseminated encephalomyelitis. In addition to neck stiffness, some support for this diagnosis comes from a consideration of the response of the patient to cortisone and prednisolone. There is evidence from the literature that cortisone or A.C.T.H. may favourably influence the course of acute disseminated encephalomyelitis. For example, Miller and Gibbons (1953) observed marked improvement in eight cases so treated. On the other hand, cortisone or A.C.T.H. appear to have little effect on the course of multiple sclerosis, a conclusion first reached by Glaser and Merritt (1952) after treating 33 chronic cases.

Of more importance is the effect of this treatment in acute cases or in those which have recently relapsed. In the small series of seven cases of multiple sclerosis reported by Miller and Gibbons each had recently developed an acute episode with some objective disability. No patient who showed any evidence of spontaneous improvement was treated. They reported that the results were "profoundly disappointing", the only definite change being a decrease in spasticity and an improvement in bladder function as previously observed by Glaser and Merritt. Alajouanine, Castaigne, and Lhermitte (1956) treated 31 cases with cortisone, 21 with an oral preparation and 10 by intrathecal injection. In 11 out of 14 patients who showed improvement, the disease was of recent origin and, with one exception, there had been a recent relapse. Neither cortisone nor A.C.T.H. had any effect in a rapidly progressive case dying within six months nor in another subacute case. In none did they observe a spectacular improvement with this form of treatment and they concluded that, when it did occur, it might have been due to a spontaneous remission. Poste and Cossa (1955) reported the case of a woman aged 20, who in the course of two years had many relapses and finally became severely disabled withe ocular pareses, severe ataxia, complete paraplegia and retention of urine. At that stage cortisone waso given in doses ranging from 100 to $200 \mathrm{mg}$. daily A.C.T.H., 10 to 20 units daily, was given during? quiescent periods. This régime was followed fo: 13 months, the patient receiving a total of 8,000 . units of A.C.T.H. The authors state that cerebella $\overrightarrow{\mathrm{F}_{3}}$ and vestibular signs cleared completely except fot nystagmus, power returned to the lower limbs, the plantar responses became flexor, and she was able to lead a normal life. Treatment was maintained byd means of a daily dose of 10 units of A.C.T.H. In a later paper, Cossa, Postel, Bochot, Camuzard, and Tartar (1956), improvement was reported in threes out of five cases of multiple sclerosis similarly. treated but they consider that this result might have్ occurred independently of treatment.

From these reports and a few others in the recent literature, it appears that, as a rule, neither cortisone nor A.C.T.H., when administered in the usual way ${ }^{N}$ has any appreciable effect on acute episodes of multiple sclerosis. Exceptionally, however, hes administration of large doses of these drugs, or $\$$ prednisolone, and their continuation in the formaf a maintenance dose may be of benefit.

In Case 1 recovery from relapses coincided the administration of prednisolone. On thre occasions when the drug was withdrawn a relapis shortly followed. Finally when she was given a maintenance dose of $10 \mathrm{mg}$. daily no relapse occurrec over a period of nine months.

Turning to the second case, the diagnosis might at first glance, appear relatively straightforward, the association of bilateral blindness with an acute myelitis favouring neuromyelitis optica. Thi $\vec{B}$ diagnosis, however, has no precise nosological status and is used somewhat indiscriminately. On the one hand, we find cases included under this heading. which, judged by the clinical and sometimes patho logical evidence, are clearly examples of multiple sclerosis, whereas in a minority the same picture may be caused by acute disseminated encephalo myelitis. In the past, undue emphasis has beerô placed on the rarity of acute retrobulbar neuritis in? this form of demyelinating disease. However, ing their recent report on nine fatal cases of encephalo $\rightarrow$ myelitis following preventive inoculation agains? rabies, Uchimura and Shiraki (1957) stress thō relative frequency of visual symptoms. In two patients demyelinating lesions were found in the optic chiasm or nerves and in three others in the optic tract or at other levels of the visual pathways 
Although in distribution and form many of the demyelinated areas resembled those of multiple sclerosis, particularly in those patients who had survived some months, the authors concluded that the essential condition was one of disseminated encephalomyelitis. It seems likely that in the Japanese race, the optic nerves and chiasm are specially vulnerable in the demyelinating diseases. For example, Okinaka, Tsubaki, Kuroira, Toyokura, Imamura, and Yoshikawa (1957) state that in 270 cases of demyelinating disease reported in Japan during the past 50 years, 175 were diagnosed as probable or possible neuromyelitis optica and this diagnosis was confirmed in each of the 18 cases which came to necropsy. In only 66 cases was the diagnosis of multiple sclerosis made or suspected. Even when allowance is made for a bias towards the diagnosis of neuromyelitis optica owing to the reputed rarity of multiple sclerosis in Japan, the above ratio is impressive, being quite the reverse of that found in the western hemisphere.

From the foregoing it is clear that acute disseminated encephalomyelitis offers an alternative explanation of the clinical picture shown by our second patient, more especially in view of the presence of neck stiffness at the onset, the associated brain-stem signs, and the response to prednisolone.

Finally, if in our two patients the primary role of herpes zoster be admitted, a further reason can be added to those already given in favour of a diagnosis of acute disseminated encephalomyelitis, namely the extreme rarity with which multiple sclerosis (or neuromyelitis optica) has followed a post-exanthematous form of encephalomyelitis.

However, our attempt to reach a precise clinical diagnosis, particularly in Case 1 , seems unnecessary in the light of a recent article by Ferraro (1958) on the subject of multiple sclerosis. He concludes that there are no appreciable differences, clinical or pathological, between acute multiple sclerosis and the acute and subacute varieties of postexanthematous, post-vaccinal, and post-infectious encephalomyelitides. Furthermore, he proposes that multiple sclerosis should be labelled as the chronic variety of disseminated encephalomyelitis. "Multiple sclerosis may then represent the chronic stage of an acute disseminated encephalomyelitis and not a transformation of a disease into another one." In support of this thesis he cites inter alia the occurrence of abortive or monosymptomatic forms of multiple sclerosis with permanent recovery, the lack of any clinical data which allow a distinction to be made between acute disseminated encephalomyelitis and acute multiple sclerosis, and the lost importance of relapses and remissions in the diagnosis of multiple sclerosis, since these may occur in various types of disseminated encephalomyelitis. While there would be general agreement with the concept, supported by Dawson in 1916, that multiple sclerosis is basically a form of encephalomyelitis, some of the reasons put forward by Ferraro for the unification of these forms of demyelinating disease are open to criticism. For example, he tends to quote exceptional cases from the literature to prove his point, while minimizing those clinical differences that are known to exist between them, for example, the comparative rarity of relapses following the post-exanthematous and postvaccinal types of acute disseminated encephalomyelitis compared with their commonplace occurrence in multiple sclerosis. It is true that cases are occasionally met with which tend to support his thesis (our first provides an excellent example), but these are comparatively rare. Ferraro's concept of an "infectious-allergic" or "toxic-allergic" pathogenesis of acute and chronic encephalomyelitis (multiple sclerosis), which is an elaboration of his earlier work (1944) on the allergic origin of the demyelinating diseases, is in keeping with recent thought on this subject.

However, until specific antigens or antibodies have been discovered, and the immunochemist has determined their inter-relationship in the various forms of encephalomyelitis, there seems little justification for the eclipse of multiple sclerosis as a clinical entity even though the term fails to describe the primary pathological feature of the disease.

\section{Summary}

A clinical account is given of acute demyelinating disease in two Japanese subjects, following an attack of herpes zoster. The first case was characterized by relapses and the second by the association of bilateral blindness with an acute myelitis. Both responded to prednisolone. They are discussed from the point of view of multiple sclerosis and acute disseminated encephalomyelitis.

We are indebted to Professor S. Okinaka and Professor F. Miyagawa for permission to publish these cases.

\section{REFERENCES}

Alajouanine, T., Castaigne, P., and Lhermitte, F. (1956). Rev. neurol., 94,352

Bogaert. L. van (1950). J. Neuropath. exp. Neurol., 9, 219. Cossa, P., Postel, J., Bochot, Camuzard, and Tartar (1956). Presse
méd., 64, 447.

Dawson, J.'W. (1916). Edinb. med. J., n.s. 17, 229.

Ferraro, A. (1944). Arch. Neurol. Psychiat. (Chicago), 52, 443.

- (1958). J. Neuropath. exp. Neurol., 17, 278.

Gordon, I. R. S., and Tucker, M. F. (1945). J. Neurol. Neurosurg. Psychiat., 8, 40 .

Glaser, G. H., and Merritt, H. H. (1952). J. Amer. med. Ass., 148 ,

Miller, H. G., and Gibbons, J. L. (1953). Brit. med. J., 2, 1345. , Stanton, J. B., and Ǵibbons, J. L. (1956). Quart. J. Med., 25,427 .

Okinaka, S., Tsubaki, T., Kuroira, Y., Toyokura, Y., Imamura, Y., and Yoshikawa, M. (1957). 'Excerpta med.' (Amst.) Sect. VIII, 10, Congress Issue.

Postel, J., and Cossa, P. (1955). Rev. neurol., 92, 286.

Uchimura, Y., and Shiraki, H. (1957). J. Neuropath. exp. Neurol., 16, 139 . 